\title{
Schizotypal personality and vulnerability to involuntary autobiographical memories
}

\section{Article}

Accepted Version

Jones, V. and Steel, C. (2012) Schizotypal personality and vulnerability to involuntary autobiographical memories. Journal of Behavior Therapy and Experimental Psychiatry, 43 (3). pp. 871-876. ISSN 0005-7916 doi: https://doi.org/10.1016/j.jbtep.2011.12.008 Available at https://centaur.reading.ac.uk/27711/

It is advisable to refer to the publisher's version if you intend to cite from the work. See Guidance on citing.

To link to this article DOI: http://dx.doi.org/10.1016/j.jbtep.2011.12.008

Publisher: Elsevier

All outputs in CentAUR are protected by Intellectual Property Rights law, including copyright law. Copyright and IPR is retained by the creators or other copyright holders. Terms and conditions for use of this material are defined in the End User Agreement.

\section{www.reading.ac.uk/centaur}

\section{CentAUR}

Central Archive at the University of Reading 
Reading's research outputs online 


\title{
Schizotypal Personality and Vulnerability to Involuntary Autobiographical Memories
}

\begin{abstract}
Background and objectives: Individuals who score high on positive schizotypy personality traits are vulnerable to more frequent trauma-related intrusive memories after a stressful event. This vulnerability may be the product of a low level of contextual integration of non-stressful material combined with a heightened sensitivity to a further reduction in contextual integration during a stressful event. The current study assessed whether high scoring schizotypes are vulnerable to frequent involuntary autobiographical memories (IAMs) of non-stressful material.
\end{abstract}

Methods: A free-association word task was used. Participants completed three recorded trials which were then replayed to allow the identification of any associations where an involuntary autobiographical memory had come to mind. Self-report measures of schizotypy and anxiety were completed.

Results: All participants retrieved at least one IAM from the three free-association word trials, with 70\% experiencing two or more IAMs. Individuals scoring high in schizotypy reported more IAMs than those who scored low. Over $75 \%$ of the memories retrieved were neutral or positive in content.

Limitations: The current study is an improvement on previous methodologies used to assess IAMs. However, bias due to retrospective recall remains a possibility.

Conclusions: Individuals scoring high in schizotypy are vulnerable to an increased level of neutral intrusive memories which may be associated with a 'baseline' level of information-processing which is low in contextual integration.

Keywords: autobiographical memory; involuntary memory; schizotypy; cognitive processing; intrusions; psychosis 


\section{Introduction}

There has been considerable recent interest in the potential relationship between traumatic life events and the presence of psychotic symptoms (Mueser, Rosenberg, Goodman, \& Trumbetta, 2002; Shevlin, Dorahy, \& Adamson, 2007). Such research indicates a high prevalence of co-morbid posttraumatic stress disorder within individuals diagnosed with schizophrenia (Kilcommons \& Morrison, 2005) and highlights areas of symptom overlap between the two disorders. Based on phenomenological observation, clinical researchers have noted the presence of involuntary intrusive memories occurring within post-traumatic stress disorder (PTSD) and psychotic disorder. Whilst vivid intrusive memories are a diagnostic criteria for PTSD (American Psychiatric Association, 1994), there is also evidence of a link between the content of intrusive memories occurring within individuals diagnosed with a psychotic disorder and the content of their psychotic symptoms (Morrison, 2001)

Steel, Fowler \& Holmes (2005) put forward a theoretical account as to how intrusive memories may develop within psychosis-prone individuals and form the basis of a psychotic episode. This account draws on the cognitive models of PTSD (Brewin, 2001; Ehlers \& Clark, 2000). These models state that intrusive memories are the product of a fundamental shift in information processing style which occurs during moments of intense fear and distress. It is argued that whilst individuals normally engage in conceptual processing (a high level of processing within which information is 'contextually integrated' with associated information of place, time etc.), there is a need to shift to a more basic and faster form of processing during a traumatic event. Consequently, there is a shift to perceptual processing (a low level form of processing based on basic perceptual information) during moments of intense distress. Whilst this shift in information processing style has a function, i.e. enabling a quick activation of an emotional response and potential action, the consequence is that the traumatic event is processed in a manner which is weak in 'contextual integration', that is the memory of the event is not integrated into a meaningful context. The result is a trauma-related memory which is hard to recall voluntarily, but is vulnerable to being triggered as an involuntary intrusive memory. Several recent studies have provided evidence for the proposed shift in processing style during a trauma (Halligan, Clark, \& Ehlers, 2002; Holmes, Grey, \& Young, 2005).

Steel et al. (2005) extend the role of contextual integration within the processing of stressful information to the concept of a full continuum, as opposed to categorical states. They argue that individual differences may occur within the baseline, or neutral information processing style that is adopted when processing non- 
emotional information. That is, individuals may vary in their strength of 'contextual integration' of incoming stimuli, such that individuals also vary in the frequency of intrusive autobiographical memories of non-distressing past events. The authors refer to the concept of schizotypal personality as a dimension which may vary in line with individual differences in 'baseline contextual integration'.

Schizotypal personality traits reflect the proposal that psychotic beliefs and experiences occur within a continuum across the non-clinical and clinical population (Claridge, 1985). Thus mild forms of hallucinations and delusions occur within individuals who score high on schizotypal personality questionnaires. High levels of these phenomena, along with many other variables, may be used to indicate psychosis-prone individuals (Woods, et al., 2009). Recent schizotypy questionnaires include subscales which enable the measurement of both positive and negative dimensions of schizotypy (O-LIFE: Mason, Claridge, \& Jackson, 1995), where positive schizotypy scales contain items related to magical thinking and unusual perceptual experiences. Individuals who score high on this subscale have been shown to perform in a similar style to individuals diagnosed with schizophrenia within a number of experimental paradigms (Steel, Hemsley, \& Jones, 1996; Tsakanikos, Sverdrup-Thygenson, \& Reed, 2003). A more recent measure, the Cardiff Anomalous Perceptions Scale (Bell, Halligan, \& Ellis, 2006) was developed to focus specifically on the unusual perceptual experiences aspects of schizotypy: this reported significant positive correlations with the Unusual Experiences subscale of the O-LIFE mentioned above.

A number of studies have been conducted which suggest that high scoring schizotypes report more frequent trauma-related intrusive memories after a stressful event than low scoring schizotypes (Holmes \& Steel, 2004; Marzillier \& Steel, 2007; Steel, Mahmood, \& Holmes, 2008).

These results suggest that high scoring schizotypes are particularly vulnerable to a change in information processing style, and an associated reduced level of contextual integration, during a stressful or traumatic event. Steel et al. (2005) also argue that high scoring schizotypes exhibit a baseline style of information processing which is lower in contextual integration that low scoring schizotypes. This difference in baseline processing style also contributes to these individuals vulnerability to engaging in very weak contextual integration during the information processing shift associated with a traumatic event.

Whilst several studies support high scoring schizotypes vulnerability to trauma-related intrusions (Holmes \& Steel, 2004; Marzillier \& Steel, 2007; Steel, et al., 2008), little is known about individual differences in 
baseline information processing style. Based on Steel et al.'s (2005) theoretical account, high scoring schizotypes will exhibit more frequent non-distressing involuntary autobiographical memories (IAMs) than low scoring schizotypes. The current study was designed to test this hypothesis.

Within memory research, IAMs are often described as "the retrieval of a specific personal episode that is brought to consciousness with apparent spontaneity" (Ball, Mace, \& Corona, 2007, p. 116), differing from 'voluntary memory' in that there is no preceding deliberate search of an individual's memory for that event. The most common methods of studying IAMs have used retrospective narratives and diary studies (Berntsen, 2009). Whilst these methods have produced a large body of research, they do have significant methodological weaknesses. The main weakness is that the participant has to be told the purpose of the task, which can easily impact on their experience of any subsequent involuntary memories. More recent methods of triggering involuntary autobiographic memories in a laboratory setting have been developed. Some of these, such as asking participants to note when they have an IAM whilst undergoing other tasks (Mace, 2006), still have the disadvantage that the participant is informed about IAMs at the start of the task, potentially leading to confusion between the deliberate and involuntary recall of autobiographical memories. However, Ball (2007) devised a paradigm which removes this problem by using a continuous free word-association task. A word association session between participant and experimenter is recorded, and subsequently played back to the participant. They are then asked to identify any moments in the free association where they had experienced an involuntary autobiographical memory (IAM).

Thus, the true purpose of the study is disguised as participants are not given any information as regards involuntary memories until after the word-association task. Ball (2007) reported that $87.5 \%$ of all trials resulted in the identification of an involuntary memory.

Using the paradigm described above (Ball, 2007) it was predicted that involuntary autobiographical memories would be more frequently triggered in high scoring schizotypes than in low scoring schizotypes. Additionally, in line with the findings from Ball (2007) it was predicted that involuntarily retrieved memories would come to mind faster and more spontaneously than memories that come to mind after a deliberate, voluntary search for autobiographical experiences, and that these involuntarily retrieved memories would contain comparable amounts of information as those memories retrieved voluntarily. 


\section{Method}

\subsection{Participants}

The total sample consisted of 49 participants ( 38 female, 11 male) with a mean age of 20 years old (s.d. 4.64, range $18-44)$. The data from one participant was excluded due to the task being interrupted. A total of 42 participants were psychology undergraduates at the University of Reading, who received course credit for taking part. The other participants were recruited from a general invitation which had been publicised locally.

\subsection{Materials}

The word stimuli were those used by Ball (2007). There were two sets of three starting words: coffee/dog/rain and popcorn/cat/thunder. Thus, both sets of words adopted the same semantic categories ('food and drink', 'pets' and 'weather-related terms').

The recording equipment consisted of a computer with an external microphone and speakers. A digital stopwatch was used to measure time to retrieval in the deliberate autobiographical memory task. Participants used two Likert scales: one to rate the spontaneity of the memory retrieval $(1=$ "came to mind spontaneously" to $7=$ "came to mind after a lot of thought") and the second to rate the amount of detail retrieved $(1=$ "recalled very few details of the experience" to $7=$ "recalled a lot of details of the experience").

\subsection{Measures}

\subsubsection{Schizotypy}

The "Cardiff Anomalous Perceptions Scale" (CAPS) (Bell, et al., 2006) is a measure of positive schizotypy which focuses on perceptual anomalies and distortions. The scale consists of 32 questions each requiring a 'Yes/No' response (range 0-32). After any positive response, follow-up questions assess three dimensions in relation to the particular experience: associated distress, intrusiveness, and frequency of occurrence for that experience. The CAPS has strong internal reliability (Cronbach's alpha $=.87$ ) and good test-retest reliability after a 6-month gap $(\mathrm{R}=0.77, \mathrm{p}<.001)$.

\subsubsection{Anxiety}

The state version of the State/Trait Anxiety Inventory (STAI) (Spielberger, Sydeman, Owen, Marsh, \& Maruish, 1999) was used. Twenty statements are rated on a four-point scale (not at all, somewhat, 
moderately, very much so) indicating how they felt at that moment in time (state anxiety). Each question is scored $1-4$, with higher scores representing higher levels of state anxiety (range 20-80). This is followed by 20 similar statements rated according to how they generally feel, i.e. trait anxiety (range 20-80). The STAI has excellent internal and test-retest reliability (Spielberger, et al., 1999).

\subsubsection{Variables measured}

For each IAM retrieved, the time the word prompting the IAM and the preceding word was recorded (this is named 'time to retrieval'). For each IAM, the participants gave a rating of spontaneity (scale of 1 'came to mind spontaneously' to 7 'came to mind after very much thought'). The participants also rated the IAM on the amount of detail retrieved (scale of 1 'recalled very few details' to 7 'recalled very many details'). Finally, the actual event being recalled within each IAM is rated for valence ('negative', 'slightly negative', 'neutral', 'slightly positive', and 'positive').

\subsection{Design}

A within-participants design was used. Word-set and the order of words within each set was counterbalanced between participants. No information was provided about involuntary memory retrievals until after the free association part of the task, in order to avoid any potential demand characteristics. There were two versions of the task (i) the involuntary retrieval task and (ii) the deliberate retrieval task. For each version, the variables recorded were the number of autobiographical memories elicited per participant, the time taken to retrieval, the content of the memory, the valence of the autobiographical experience and the participant's ratings of spontaneity and detail of retrieval.

\subsection{Procedure}

After providing informed consent, demographic details were obtained and participants were given instructions for the involuntary retrieval part of the exercise, i.e. the "Word-Association Task". A 'starting word' (e.g. computer) was announced by the researcher, to which the participant was told to respond with the first word that came to mind. Their response was then repeated by researcher, the participant then generated a new response, and this word-association was continued in sequence for around 30 seconds.

A practice task, using the starting word 'computer', was completed and if the participant understood the task and produced a chain of words for 20 seconds, audio-recording commenced and the three experimental trials took place. Once all three trials were completed, the responses were then replayed and the participant 
was told to think about what had been in their mind when they had made these responses. They were asked to identify any point that a personal experience had been in their mind: the definition of a 'personal experience' was explained by the researcher as 'the recall of an event or experience from your own past or information and knowledge personal to you". For identified experiences, the participant provided a brief description and rated their memory of the experience on scales of spontaneity of retrieval and on the amount of details retrieved, plus an indication of the valence of the event. This was repeated for all three trials. The first memory retrieved in each word-association trial was identified and subjected to further assessment. Thus, with the starting word of RAIN, the word chain could be "RAIN - fall - accident emergency" with an associated memory of visiting friend in hospital, passing A\&E in the rain.

The deliberate retrieval task was then conducted: the "Word Cue-Task", which involved intentional recall of autobiographical events. The participant was given a cue word and told to bring voluntarily to mind an autobiographical memory in relation to that word only. So, for example, with the cue word "RAIN", the deliberately retrieved memory the participant described could be "getting caught in a rainstorm on holiday in Florida". There were three word-cued trials, with the three cue words matching the starting words used in the word-association trials. For each memory, the time between the starting word and the retrieval was recorded, along with a brief description of the experience, valence of experience, rating of spontaneity, and rating of amount of details retrieved.

After the completion of the experimental tasks, the participant was asked to complete the two self-report measures described above (CAPS, STAI). They then completed another cognitive task, the results of which are not reported here. Finally, the participant was debriefed and given an opportunity to ask questions about the study and given information about potential sources of support in case they later became distressed.

\subsection{Statistical Analysis}

Participants were divided into high and low scoring schizotype groups by employing a median split on the total CAPS score. The inclusion of the STAI allowed for analyses to consider state and trait anxiety as a potential covariate. For these analyses participants were divided into high and low scoring state anxiety groups, based on a median split.

Regressions and ANCOVAs were used to examine the relationships between anxiety and schizotypy. Mann-Whitney tests (non-parametric) were used to examine frequencies of both IAMs and deliberate 
memories by schizotype group and by anxiety group. A Wilcoxon Signed Rank test was run on the paired variable measuring the frequency of memory retrieval. Paired t-tests were computed to compare the equivalent variables from the two tasks (involuntary retrieval and voluntary retrieval). 


\section{Results}

\subsection{Schizotypy}

The mean score on the CAPS was 9.6 (s.d. =5.1), the median was 9.5 (range 0-21). The total CAPS score was used to divide the participants into two groups: Low Schizotypy (scored under 10) and High Schizotypy (scored 10 or above). This compares to the original data on the CAPS (Bell, et al., 2006) which reported a mean of $7.3(\mathrm{~s} . \mathrm{d}=5.8)$.

There was a mean trait anxiety score of 41.04 (s.d.=11.37). The low trait anxiety group were those scoring under 41 , and the high trait anxiety group those who scored 41 or more. There was a mean state anxiety score of 32.85 (s.d.=9.10). The low state anxiety group were those scoring under 33, and the high state anxiety group those who scored 33 or more.

The low and high scoring schizotypy groups did not significantly differ in terms of trait anxiety $(\mathrm{t}(46)=1.438, \mathrm{p}=0.16)$, state anxiety $(\mathrm{t}(46)=0.806, \mathrm{p}=0.42)$, age $(\mathrm{t}(46)=1.413, \mathrm{p}=0.16)$ or gender $(\mathrm{t}(46)=$ $0.211, \mathrm{p}=0.83)$.

\subsection{Word-Association (involuntary memories) Condition}

\subsubsection{Frequency of Involuntary Autobiographical Memories (IAMs)}

Overall 101 trials (70\%) elicited an IAM. All participants retrieved an autobiographical memory which related to their word-association stimuli in at least one of the three trials. There was no significant difference in the number of participants retrieving 1, 2 or 3 IAMS (14 participants retrieved one IAM, 15 two IAMs and 19 three IAMs; $\left.\left(\chi^{2}(2)=0.87, p=0.65\right)\right)$. There was no significant relationship between number of IAMs produced and gender $\left(\chi^{2}(2)=3.30, \mathrm{p}=0.19\right)$ or age $\left(\chi^{2}(2)=2.25, \mathrm{p}=0.32\right)$.

\subsubsection{IAMs and Schizotypy}

Participants in the high schizotypy group produced more IAMs (within the three trials) than those in the low schizotypy group $(\mathrm{U}=173, \mathrm{z}=2.52$, $\mathrm{p}=0.01$, effect size $\mathrm{r}=0.36)$ as shown in Table 1 . Anxiety was not significantly related to IAM frequency (trait anxiety $\mathrm{U}=285, \mathrm{z}=.077, \mathrm{p}=0.94$; state anxiety $\mathrm{U}=279, \mathrm{z}=$ $.187 \mathrm{p}=0.85)$.

INSERT TABLE 1 HERE 
The mean number of word associations made by participants before an IAM was retrieved within any one trial was 3.67 (s.d. =1.58). There was no significant difference between high (mean =3.95 (s.d. $=1.59)$ and low scoring schizotypes $($ mean $=3.40($ s.d. $=1.55)(t(46)=1.43, \mathrm{p}=0.16)$ regarding the number of word associations made. There was no significant difference in the mean length of time to retrieve an IAM between high $($ mean $=2.7 \mathrm{sec}($ s.d. $=1.8)$ and low scoring schizotypes $($ mean $=2.3 \sec (\mathrm{s} . \mathrm{d} .=0.48)(\mathrm{t}(46)=$ $1.14, \mathrm{p}=0.26)$.

\subsubsection{Data analysis by individual trial:}

Data analysis was conducted to explore whether there were any underlying differences in the characteristics of the trials that resulted in the retrieval of an IAM ('Yes' trials) and those trials which did not ('No' trials). In each trial, the total number of responses made was recorded: the totals ranged between 6 and 14, with a mean of 9.6 responses (s.d. $=1.6)$. 'Yes' trials had a mean number of responses of $9.7($ s.d. $=1.6)$, compared to a mean of $9.5($ s.d. $=1.8)$ for 'No' trials. The difference was not statistically significant: $F(1,142)=0.80$, $\mathrm{p}=0.37$.

Additionally, on each trial the times between each response were recorded producing a mean response time per trial (range $1.55 \mathrm{sec}$ to $4.98 \mathrm{sec}$ ). The 'Yes' trials had a mean of 2.83 (s.d. = 0.45), and 'No' trials had a mean of 2.95 (s.d. $=0.60$ ): again, this difference was not statistically significant: $F(1,142)=1.66, p=0.20$ ).

Trials were analysed with reference to the starting word, to investigate whether any particular category or word produced more IAMs: chi-square analyses showed no statistically significant differences (for category, $\chi^{2}(2)=1.260, \mathrm{p}=0.533$, and for word $\left.\chi^{2}(5)=1.757, \mathrm{p}=0.882\right)$.)

\subsubsection{IAMs retrieved and emotional valence}

Each IAM was rated for valence: Negative (6\% of all IAMs), Slightly Negative (19\%), Neutral (43\%), Slightly Positive (30\%) or Positive (3\%). There were no significant differences between valence category and ratings of spontaneity $\left(\chi^{2}(20)=10.66, p=0.956\right)$ or detail $\left(\chi^{2}(20)=18.06, p=0.58\right)$. There was no significant difference within the frequency of distribution of the IAM valence category and high and low schizotype group $(\chi 2(4)=2.68, p=0.61)$. 


\subsection{Word-cued (deliberate autobiographical memories) Condition}

In the word-cued task 137 trials (95\%) led to a deliberately retrieved autobiographical memory, with $88 \%$ of participants retrieving a memory for each of the three cued words, and every participant retrieving a memory for at least one trial.

\subsubsection{Differences between the involuntary and voluntary memory retrievals}

A comparison of the number of autobiographical memories retrieved in each of the tasks was made. Participants recalled significantly more memories with the deliberate retrieval task, $($ mean $=2.85)$ than involuntarily with the involuntary retrieval task $($ mean $=2.10)(\mathrm{z}=4.343, \mathrm{~N}-\mathrm{Ties}=30, \mathrm{p}<0.001)$.

Analyses between deliberate and involuntary retrievals for other variables of interest were conducted. Memory recall was faster for involuntary autobiographical memories than for deliberately recalled autobiographical memories $(\mathrm{t}(47)=5.69, \mathrm{p}<0.001)$. The rating of spontaneity was significantly faster in the involuntary retrieval task $(\mathrm{t}(47)=3.61, \mathrm{p}<0.001)$. There was no significant difference between the two memory types in the participants' rating of the detail recalled $(\mathrm{t}(47)=0.63, \mathrm{p}=0.20)$.

\subsubsection{Comparison between schizotype groups for involuntary/voluntary memories}

A 'difference' variable was created in order to compare the number of autobiographical memories retrieved in the two tasks. This was computed by deducting the number of memories retrieved involuntarily from the number of memories retrieved deliberately (DAM-IAM). As shown in Table 2, these differences ranged from -1 (1 more IAM than DAM), 0 (no difference between numbers of IAMs retrieved and DAMs retrieved),+1 (I more DAM than IAM) and +2 (2 more DAMs than IAMS). The DAM-IAM mean was almost significantly lower in the high schizotype group $(\mathrm{U}=198, \mathrm{z}=1.955, \mathrm{p}=0.051$, effect size $\mathrm{r}=0.28)$ but did not approach significance within the anxiety groups (trait anxiety: $U=278, z=0.29, p=0.82$; state anxiety: $\mathrm{U}=26, \mathrm{z}=0.26, \mathrm{p}=0.79$ ).

\section{INSERT TABLE 2 HERE}




\section{Discussion}

Individuals who scored high in schizotypy reported more frequent non-distressing involuntary autobiographical memories. This result supports the hypotheses based on the theoretical account put forward by Steel et al. (2005). The cognitive model of trauma-related intrusions (Steel, Fowler, \& Holmes, 2005) suggests that an individual scoring high on schizotypal traits would be more vulnerable to developing intrusions due to a baseline processing style which is relatively weak in contextual integration (CI). Previous studies have shown that high schizotypes are vulnerable to intrusive trauma-related memories after a stressful or traumatic event (Holmes \& Steel; 2004; Marzillier \& Steel, 2007; Steel, Mahmood \& Holmes, 2008). However, the current study is the first to report high scoring schizotypes vulnerability to more frequent involuntary memories of non-stressful material, thus supporting the role of reduced 'baseline' levels of contextual integration within this group.

The specificity of this finding is enhanced by the fact that high scoring schizotypes did not differ from low scoring schizotypes as regards the frequency of deliberately retrieved memories. Further, there were no significant differences within the self-report ratings on the amount of detail or spontaneity of recall of the IAMs between high and low scoring schizotypy groups. In support of Ball (2007), the current results showed that IAMs came to mind more quickly than memories after a deliberate, voluntary search, and participants rated the involuntary retrieved memories as 'more spontaneous' than the deliberately retrieved memories. There were no differences in characteristics (such as number of words in association chain, mean time taken per association) between the trials that elicited IAMs and those that did not. All participants reported at least one IAM, and over $75 \%$ of IAMs were of neutral or positive content suggesting that we were not recording intrusive trauma-related memories. These results support the validity of using the current experimental paradigm as a way of triggering involuntary memories. Although the current study did not reveal any significant differences in anxiety between low and high scoring schizotypes, analyses between anxiety and IAMs were conducted given previous reports of a relationship between anxiety and schizotypy (Steel, Marzillier, Fearon, \& Ruddle, 2009). There were no significant differences between the low and high anxiety groups on the number of IAMs retrieved, indicating that the vulnerability to more frequent IAMs was distinct to schizotypy and not associated with anxiety. 
The word-association task was simple to carry out and effective in triggering involuntary memories, indicating the potential for use within clinical populations. Whilst the participants were mostly young undergraduate psychology students, the schizotypy scores recorded were comparable to reported norms and represented a range of scores, suggesting the results may be generalizable.

However, it is possible that the memories retrieved may not all be involuntary. Participants were not asked to identify IAMs until after they have completed the three word-chaining trials, when they listened to a recording of the word chains. It is therefore difficult to verify the participants report that the IAM had occurred during the original word association task, or whether they had falsely recalled this experience. A further limitation is that the current study did not consider participant's previous exposure to traumatic events in relation to IAMs. Future research is needed to investigate whether a history of trauma has an impact on an individual's vulnerability to IAMs of neutral content as well as intrusive trauma-related memories. Studies using this paradigm within clinical populations (such as participants diagnosed with PTSD) will provide data to allow us to address this and other questions.

This paradigm was chosen to obtain spontaneously retrieved involuntary autobiographical memories, providing data to assess the relationship between vulnerability to the triggering of memories and individuals' schizotypy level. Whilst we argue that the completion of this task is associated with the process of 'baseline' contextual integration, the memory retrieval component may be associated with other aspects of cognitive processing. These may include heightened activation triggered by the free association cuing, which would be in line with the reduced cognitive inhibition displayed in high schizotypes (Steel, et al., 1996).

The presence of more frequent intrusive memories has been argued to be the basis of a psychotic experience for some individuals (Steel et al., 2005). This account highlights that high scoring schizotypes are not just vulnerable to more frequent intrusions, but also more vulnerable to making 'source monitoring' errors (Larøi, Collignon, \& Van der Linden, 2005). That is, these individuals are more likely to confuse an internally generated event (such as a memory) with externally generated events (having seen or heard something). Such errors may account for experiences such as 'déjà vu', which may be an intrusive memory which is not identified as such, and therefore experienced as a strong sense of familiarity without context. Steel et al. (2005) go on to argue that such confusing experiences will be understood with reference to an individual's belief system. For high scoring schizotypes, these beliefs often include clairvoyance and 
telepathy and a 'déjà vu' experience is more likely to be interpreted within a paranormal framework. Such interpretations may become more threatening when the content of the confusing intrusive memories are more threatening. For example, memories of a past assault may be interpreted as a premonition of a future assault. If the source of these experiences is attributed to the Devil, God or government agencies, this presentation may appear to be consistent with a psychotic episode. Whilst the evidence is growing in relation to the intrusion vulnerability aspect of Steel et al.'s (2005) account, further research is required to explore high scoring schizotypes appraisal of such intrusions.

Whilst intrusive memories within psychosis are often associated with negative affect, there may be some advantages to the underlying processes within high scoring schizotypes. For example, easily triggered involuntary memories may enable individuals to access episodic information with little cognitive effort, which may provide an increased sense of continuity, extending an individual's 'temporal horizon' (Rasmussen \& Berntsen, 2009). Also, high scoring schizotypes show an increased ability for mental 'time travel', that is where an individual can mentally imagine themselves back in time to relive events, or forwards in time to pre-live events (Winfield \& Kamboj, 2010).

In summary, the current results suggest that positive schizotypal personality traits are associated with a vulnerability to more frequent involuntary autobiographical memories. The word-association paradigm has proved a useful tool which could be employed within future clinical studies. 


\section{$\underline{\text { References }}$}

American Psychiatric Association, A. (1994). Diagnostic and Statistical Manual for Mental Disorders. In (4th ed.). Washington, DC: APA.

Ball, C. T. (2007). Can we elicit involuntary autobiographical memories in the laboratory? In J. H. Mace (Ed.), Involuntary memory (pp. 127-152). Oxford: Blackwell Publishing.

Ball, C. T., Mace, J. H., \& Corona, H. (2007). Cues to the Gusts of Memory. In J. H. Mace (Ed.), Involuntary memory (pp. 113-126). Oxford: Blackwell Publishing.

Bell, V., Halligan, P. W., \& Ellis, H. D. (2006). The cardiff anomalous perceptions scale (CAPS): A new validated measure of anomalous experience. Schizophrenia Bulletin, 32, 366-377.

Berntsen, D. (2009). Involuntary autobiographical memories: An introduction to the unbidden past. New York, NY US: Cambridge University Press.

Brewin, C. R. (2001). A cognitive neuroscience account of posttraumatic stress disorder and its treatment. Behaviour Research and Therapy, 39, 373-393.

Claridge, G. S. (1985). Origins of mental illness: Oxford: Blackwell.

Ehlers, A., \& Clark, D. M. (2000). A cognitive model of posttraumatic stress disorder. Behaviour Research and Therapy, 37, 319-345.

Halligan, S. L., Clark, D. M., \& Ehlers, A. (2002). Cognitive processing, memory, and the development of PTSD symptoms: two experimental analogue studies. Journal of Behavior Therapy and Experimental Psychiatry, 33, 73-89.

Holmes, E. A., Grey, N., \& Young, K. A. D. (2005). Intrusive images and 'hotspots' of trauma memories in Posttraumatic Stress Disorder: An exploratory investigation of emotions and cognitive themes. Journal of Behavior Therapy and Experimental Psychiatry, 36, 3-17.

Holmes, E. A., \& Steel, C. (2004). Schizotypy: A Vulnerability Factor for Traumatic Intrusions. Journal of Nervous and Mental Disease, 192, 28-34.

Kilcommons, A. M., \& Morrison, A. P. (2005). Relationships between trauma and psychosis: an exploration of cognitive and dissociative factors. Acta Psychiatrica Scandanavia, 112, 351-359.

Larøi, F., Collignon, O., \& Van der Linden, M. (2005). Source monitoring for actions in hallucination proneness. Cognitive Neuropsychiatry, 10, 105-123.

Mace, J. H. (2006). Episodic remembering creates access to involuntary conscious memory: Demonstrating involuntary recall on a voluntary recall task. Memory, 14, 917-924.

Marzillier, S. L., \& Steel, C. (2007). Positive Schizotypy and Trauma-Related Intrusions. Journal of Nervous and Mental Disease, 195, 60-64.

Mason, O., Claridge, G., \& Jackson, M. (1995). New scales for the assessment of Schizotypy. Personality and Individual Differences, 18.

Morrison, A. P. (2001). The interpretation of intrusions in psychosis: An integrative cognitive approach to hallucinations and delusions. Behavioural and Cognitive Psychotherapy, 29, 257-276.

Mueser, K. T., Rosenberg, S. D., Goodman, L. A., \& Trumbetta, S. L. (2002). Trauma, PTSD, and the course of schizophrenia: An interactive model. Schizophrenia Research, 53, 123-143.

Rasmussen, A. S., \& Berntsen, D. (2009). The possible functions of involuntary autobiographical memories. Applied Cognitive Psychology, 23, 1137-1152.

Shevlin, M., Dorahy, M. J., \& Adamson, G. (2007). Trauma and Psychosis: An Analysis of the National Comorbidity Survey. American Journal of Psychiatry, 164, 166-169.

Spielberger, C. D., Sydeman, S. J., Owen, A. E., Marsh, B. J., \& Maruish, M. E. (1999). Measuring anxiety and anger with the State-Trait Anxiety Inventory (STAI) and the State-Trait Anger Expression Inventory (STAXI). In The use of psychological testing for treatment planning and outcomes assessment (2nd ed.). (pp. 993-1021). Mahwah, NJ US: Lawrence Erlbaum Associates Publishers.

Steel, C., Fowler, D., \& Holmes, E. A. (2005). Trauma-Related Intrusions and Psychosis: An Information Processing Account. Behavioural and Cognitive Psychotherapy, 33, 139-152.

Steel, C., Hemsley, D. R., \& Jones, S. (1996). Cognitive Inhibition and Schizotypy as measured by the OxfordLiverpool Inventory of Feelings and Experiences. Personality and Individual Differences, 20, 769-773.

Steel, C., Mahmood, M., \& Holmes, E. A. (2008). Positive schizotypy and trait dissociation as vulnerability factors for post-traumatic distress. British Journal of Clinical Psychology, 47, 245-249.

Steel, C., Marzillier, S., Fearon, P., \& Ruddle, A. (2009). Childhood abuse and schizotypal personality. Social Psychiatry and Psychiatric Epidemiology, 44, 917-923. 
Tsakanikos, E., Sverdrup-Thygenson, L., \& Reed, P. (2003). Latent inhibition and psychosis-proneness: visual search as a function of pre-exposure to the target and schizotypy level. Personality and Individual Differences, 34, 575-589.

Winfield, H., \& Kamboj, S. K. (2010). Schizotypy and mental time travel. Consciousness and Cognition, 19, 321327.

Woods, S. W., Addington, J., Cadenhead, K. S., Cannon, T. D., Cornblatt, B. A., Heinssen, R., Perkins, D. O., Seidman, L. J., Tsuang, M. T., Walker, E. F., \& McGlashan, T. H. (2009). Validity of the prodromal risk syndrome for first psychosis: Findings from the North American Prodrome Longitudinal Study. Schizophrenia Bulletin, 35, 894-908. 
Table 1: Number of word-association trials eliciting an IAM, by schziotypy and anxiety groups $\%$ of group retrieving:

Mean (s.d.)

$\mathrm{n}$ number of IAMs One IAM Two IAMs Three IAMs

\begin{tabular}{lcccccc}
\hline $\begin{array}{l}\text { Schizotypy } \\
\text { group: }\end{array}$ & High & 24 & $2.42(0.65)$ & $8.33 \%$ & $41.67 \%$ & $50.00 \%$ \\
& Low & 24 & $1.79(0.88)$ & $50.00 \%$ & $20.83 \%$ & $29.17 \%$ \\
\hline $\begin{array}{l}\text { Trait Anxiety } \\
\text { group: }\end{array}$ & High & 24 & $2.13(0.74)$ & $20.83 \%$ & $45.83 \%$ & $33.33 \%$ \\
\hline $\begin{array}{l}\text { State Anxiety } \\
\text { group: }\end{array}$ & Low & 24 & $2.08(0.93)$ & $37.50 \%$ & $16.67 \%$ & $45.83 \%$ \\
\hline
\end{tabular}

Table 2: Frequency Difference by Group

\section{Frequency Difference: DAMs - IAMs}

\begin{tabular}{|c|c|c|c|c|c|c|}
\hline & & -1 & $\mathbf{0}$ & 1 & 2 & Mean (s.d.) \\
\hline \multirow{2}{*}{$\begin{array}{l}\text { Schizotypy } \\
\text { group: }\end{array}$} & High & 2 & 6 & 6 & 10 & 1.00 \\
\hline & Low & 1 & 12 & 9 & 2 & 0.50 \\
\hline \multirow{2}{*}{$\begin{array}{l}\text { Trait Anxiety } \\
\text { group: }\end{array}$} & High & 2 & 8 & 9 & 5 & $0.71(0.91)$ \\
\hline & Low & 1 & 10 & 6 & 7 & $0.79(0.93)$ \\
\hline \multirow{2}{*}{$\begin{array}{l}\text { State Anxiety } \\
\text { group: }\end{array}$} & High & 1 & 8 & 9 & 5 & $0.78(0.85)$ \\
\hline & Low & 2 & 10 & 6 & 7 & $0.72(0.98)$ \\
\hline
\end{tabular}

